\title{
WILEY-VCH
}

DOI: $10.1002 /$ ((please add manuscript number))

Article type: Full Paper

\section{Hybrid plasmonic cavity modes in arrays of gold nanotubes}

Junfeng Wang, Cheng Zhang, Junxi Zhang*, Haojie Song, Pei Wang, Yonghua Lu, Guangtao Fei, Wen Xu, Wei Xu, Lide Zhang, Yuri S. Kivshar, and Lin Zhang

J. Wang, Dr. J. Zhang, H. Song, Dr. G. Fei, W(ei). Xu, Prof. L. D. Zhang

Key Laboratory of Materials Physics

Anhui Key Laboratory of Nanomaterials and Nanostructures

Institute of Solid State Physics

Chinese Academy of Sciences

Hefei 230031, P. R. China

E-mail: jxzhang@issp.ac.cn

C. Zhang, Prof. P. Wang, Dr. Y. Lu

Department of Optics and Optical Engineering

University of Science and Technology of China

Hefei 230026, P. R. China

Dr. J. Zhang, Prof. L. Zhang

Aston Institute of Photonic Technologies

School of Engineering \& Applied Science

Aston University

Birmingham B4 7ET, UK

Dr. W(en). Xu

Research Laboratory of Computational Materials Sciences

Institute of Solid State Physics

Chinese Academy of Sciences

Hefei 230031, P. R. China

Prof. Y. S. Kivshar

Nonlinear Physics Centre

Research School of Physics and Engineering

Australian National University

Canberra ACT 2601, Australia

Keywords: plasmonic resonator, cavity mode, hybrid resonance mode, gold nanotube, array 


\section{WILEY-VCH}

Plasmonic structures are known to confine light at the nanometer scale, and they exhibit enhanced electromagnetic fields localized in small mode volumes. Here we study plasmonic resonators based on a metamaterial consisting of periodic arrays of gold nanotubes embedded into anodic aluminum oxide and demonstrate strong confinement of local fields with low losses. We observe higher-order resonance modes of surface plasmons localized in gold nanotubes when the nanotube length exceeds some critical values. Our numerical simulations suggest that for the higher-order modes, the electric fields are mainly localized at the interfaces between aluminum oxide and gold in the form of the standing-wave longitudinal plasmonic modes, partially localized in the pores and at two ends of the nanotubes owing to the strong coupling of the Fabry-Pérot resonances with extraordinary optical transmission in the periodical structures through the inner nanochannels of the nanotubes, so that the nanotubes play a role of efficient cavity resonators. We reveal the existence of hybrid resonant cavity modes with asymmetrical distributions of the electric field resulting from the near-field coupling of both transverse and longitudinal modes in the gold nanotube metamaterials. 


\section{WILEY-VCH}

\section{Introduction}

Realization of strong light-matter interaction is the fundamental problem of nanophotonics that aims designing subwavelength optical components. ${ }^{[1]}$ Enhancement of the light-matter coupling can be achieved by using resonant cavities with high Purcell factors. ${ }^{[2]}$ Enhancing the Purcell factor, which is directly proportional to the cavity quality factor Q and inversely proportional to the mode volume $\mathrm{V}$, can be achieved by either increasing $\mathrm{Q}$ or decreasing $\mathrm{V} .{ }^{[3]}$ In general, dielectric cavities based on Fabry-Pérot resonators, whispering gallery modes, and photonic crystals demonstrate very high values of $\mathrm{Q},{ }^{[4]}$ but their modal volumes are large. For the conventional dielectric cavities, reducing the modal volumes or physical sizes to the nanometer scale is restricted by the diffraction limit of light. Moreover, in many cases, for a relatively small modal volume, the optical modes of conventional cavities cannot match the modes to form the plasmon polaritons required by momentum and energy conservation for the efficient coupling. In contrast, plasmonic resonant cavities are capable of confining light at the nanometer scale, which leads to both enhanced local electromagnetic fields ${ }^{[5-9]}$ and low mode volumes, ${ }^{[1,9-12]}$ and suggest promising applications for subwavelength optics and nanolasers, ${ }^{[13-16]}$ nanoantennas, ${ }^{[17-19]}$ sensing, ${ }^{[20-23]}$ enhanced nonlinear effects, ${ }^{[18,24-27]}$ Surface enhanced Raman scattering, ${ }^{[8]}$ and solar cell elements, ${ }^{[28,29]}$ to name a few.

Since Ditlbacher et al. demonstrated plasmonic resonators based on silver nanowires, ${ }^{[30]}$ metal nanorod-types structures have been exploited extensively for a design of plasmonic resonators. ${ }^{[30-36]}$ In such structures, surface plasmon polaritons (SPPs) excited at the metaldielectric interfaces are reflected backward and forward between the metal nanostrip terminations resulting in Fabry-Pérot resonances. ${ }^{[30,32,33]}$ Although the metal nanostrips have very small mode confinement, very large Ohmic losses occur at the single metal-dielectric interface of the metal nanostrips. In this case, the enhancement of mode coupling through engineering of multiple metal-dielectric interfaces could lead to a reduction of the Ohmic losses. When two metal-dielectric interfaces are close to each other, the coupling between 


\section{WILEY-VCH}

SPPs at the two interfaces gives rise to antisymmetric and symmetric modes, which correspond to the short range surface plasmons (SRSP) or slow SPP modes and long-range plasmons (LRSP) or fast SPP modes. ${ }^{[19,34]}$ When the metal nanostrips bounded on two symmetric dielectric layers support the short-range and long-range SPP modes, the dielectric layer embedded in two metal nanostrips supports the gap plasmon (long-range SPP) modes. ${ }^{[37-}$

${ }^{43]}$ The SRSP mode is very strongly bound to the metal strips and the LRSP mode is weakly confined to the metal strips. As the consequence, the former is characterized by larger Ohmic losses. Hence, the trade-off between localization and loss is crucial to design good-quality plasmonic resonant cavities. ${ }^{[44]}$ Generally, it is difficult to obtain simultaneously very low losses and extremely strong confinements of electric fields in the plasmonic resonators based upon individual structures covering the traditional insulator-metal-insulator (IMI) and metalinsulator-metal (MIM) geometries.

In this paper, we suggest a novel type of plasmonic resonators based on periodic coupling of arrays of gold nanotubes embedded in anodic aluminum oxide (AAO). We notice that gold nanotubes demonstrate versatile performances for the plasmonic resonators. Beside the advantage of strong coupling at periodic metal-dielectric interfaces in the structures such as gold nanorods and nanowire arrays, ${ }^{[45-47]}$ the gold nanotubes with a hollow geometry are similar to the subwavelength hole arrays which exhibit the effect of extraordinary optical transmission ${ }^{[48-50]}$ leading to sharper Fabry-Pérot resonances originating from the reflections at the nanotube terminations. In this case, the metamaterials composed of an array of gold nanotubes becomes a promising candidate for plasmonic resonators since it allows to achieve both strong confinement of electric fields at the nanotube walls with low losses. On the other hand, the measurement of the reflectance suggests that the longitudinal resonance exhibits high-order modes, and the number and frequency of the longitudinal modes can be tailored by tuning the nanotube length. Furthermore, the electric field distributions of the plasmonic resonators simulated by using FDTD demonstrate that the electric fields at the surfaces of the 


\section{WILEY-VCH}

gold nanotube have standing-wave patterns for the longitudinal resonance modes with different orders. Moreover, the harmonics of the standing waves with odd and even orders can be excited for the different nanotube lengths, and the harmonic modes can be selected by varying the nanotube length. We observe asymmetric T-L hybrid plasmonic modes originating from the coupling of transversal and longitudinal resonances, which depend on the structure parameters of the gold nanotube arrays. The proposed plasmonic resonators can provide fundamental background for novel nanophotonic devices and efficient sensing of organic molecules.

\section{Results and discussion}

\subsection{Assembly and characterization of plasmonic gold nanotube arrays}

Gold nanotube arrays are synthesized successfully by using a galvanostatic electrodeposition process into the nanochannels of the AAO membranes, meanwhile, the controllable formation of the gold nanotubes strongly depends on the thickness of the gold film as the working electrode with tube-like framework geometry (Figure 1 a), the constant current based on a chronopotentiometry technique action, and the electrodeposition time. Figure $1 \mathrm{~b}$-f shows the deposition potential with the time during the electrodeposition of the gold nanotube arrays when the current is fixed to $-0.5 \mathrm{~mA}$ ( $\mathrm{vs} \mathrm{Ag} / \mathrm{AgCl})$. It can be seen that the deposition potential first dramatically declines and then tends to be stable, which results from the first nucleation and the subsequent growth processes of the gold nanotubes. Moreover, the length of the $\mathrm{Au}$ nanotubes can be tuned by changing the electrodeposition time from $500 \mathrm{~s}, 600 \mathrm{~s}, 900 \mathrm{~s}, 1600$ s, and to 1900 s. Figure 2 a-e shows the SEM images of the Au nanotube arrays embedded in the AAO membranes with the same diameter (e.g., inner diameter $=20 \mathrm{~nm}$, outer diameter $=$ $55 \mathrm{~nm}$ ) but different lengths from $205 \mathrm{~nm}, 300 \mathrm{~nm}, 390 \mathrm{~nm}, 440 \mathrm{~nm}$ to $670 \mathrm{~nm}$ (The length distribution histograms has shown in the supporting information Figure S1), which 


\section{WILEY-VCH}

correspond to the electrodeposition time of $500 \mathrm{~s}, 600 \mathrm{~s}, 900 \mathrm{~s}, 1600 \mathrm{~s}$, and $1900 \mathrm{~s}$, respectively.

\subsection{Measurements of plasmonic cavity modes of gold nanotube arrays}

The reflectance measurements indicate the cavity modes of LSPs in the Au nanotube arrays can be tailored by the length of the nanotubes (Figure 3 a-e), the multiple resonance peaks appear when the nanotube length changes. The peaks marked with $\mathrm{T} 1$ and $\mathrm{T} 2$ for all of the nanotube arrays have the same resonance wavelengths of about $490 \mathrm{~nm}$ and $430 \mathrm{~nm}$, which correspond the two transversal resonance modes associated with the outer and inner diameter of the nanotubes. Furthermore, the peaks marked with $\mathrm{Li}(i=1,2,3$ and 4) represent the highorder modes of the longitudinal resonance of LSPs which are strongly dependent on the length of the nanotubes. The resonance mode L1 demonstrates distinct red-shifts from $828 \mathrm{~nm}$, $930 \mathrm{~nm}$, and $1314 \mathrm{~nm}$ to $1581 \mathrm{~nm}$ with increasing the nanotube length from $205 \mathrm{~nm}$ to 440 $\mathrm{nm}$. It is has been noted that a longitudinal resonance mode L2 appears around $763 \mathrm{~nm}$ when the nanotube length increases to $390 \mathrm{~nm}$, additionally, the mode L2 redshifts from $763 \mathrm{~nm}$ to $864 \mathrm{~nm}$ with raising the nanotube length from $390 \mathrm{~nm}$ to $440 \mathrm{~nm}$. It is noting that the highorder harmonics of the longitudinal resonance starts with the resonance mode L2 at $1860 \mathrm{~nm}$ and subsequently appears the mode L3 at $1060 \mathrm{~nm}$ and L4 at $750 \mathrm{~nm}$ from right to left when the nanotube length increases to $670 \mathrm{~nm}$, which exhibits different characteristics compared with those nanotubes with the lengths from $205 \mathrm{~nm}$ to $440 \mathrm{~nm}$. These results can be explained by the following theoretical simulation. These experimental measurements indicate that the resonance modes with the same orders red shifts with increasing the nanotube length, and the resonance mode with different orders appears when the nanotube enhances to those specific lengths (e.g., $390 \mathrm{~nm}$ and $670 \mathrm{~nm}$ ). 


\section{WILEY-VCH}

\subsection{Simulation of plasmonic cavity modes and electric field distributions of gold}

nanotube arrays

On the other hand, the resonance mode and their electric field distributions of LSPs in the gold nanotube arrays embedded in the AAO membranes can be simulated by using FDTD method under the perfect matched layer (PML) condition. TE-polarized light was used to simulate reflectance spectra since resonant extinction is similar to those observed in the reflectance measurements. The refractive index of the AAO membranes is $1.61 .^{[51]}$ Figure 4 a-d illustrate the reflectance spectra and the electric field distributions of the plasmonic resonance modes in the gold nanotube array of $205 \mathrm{~nm}$ in length. The FDTD simulation indicates both the transverse and longitudinal resonance modes perform very strong confinements of electric fields on the interfaces. The electric field of the resonance mode marked with T2 is concentrated on the incident ends of the gold nanotubes (Figure 4b), so the mode $\mathrm{T} 2$ is one of the transversal resonance modes. While, the electric fields of the longitudinal resonance mode L1 mainly focuses on the outer surfaces of the gold nanotubes (that is the interfaces between the aluminum oxide and the nanotubes), and the electric fields on the surface of the gold nanotubes perform a standing-wave mode (Figure 4d), this is originated from the Fabry-Pérot resonance because of the back and forth reflections between the terminations of the gold nanotubes, which exhibits the characteristics of a cavity resonator. Moreover, the electric fields of the longitudinal resonance mode L1 is partially localized in the pores of the gold nanotubes (Figure 4d), indicating the extraordinary optical transmission coupling effect based on the periodical hollow structures in the gold nanotube arrays can distinctly enhance the Fabry-Pérot resonance between the top and the bottom terminations of the metal nanotubes, which gives rise to the low losses in the plasmonic resonator. It is interesting to note that the electric fields of the resonance mode $\mathrm{T} 1$ are not only located at the incident ends of the nanotubes but also distributed on the interfaces between the aluminum oxide and the nanotubes (the outer walls) as well as the interfaces between the air (pores) and 


\section{WILEY-VCH}

the nanotubes (the inner walls), that is, the resonance mode T1 covers both the transversal and the longitudinal resonance modes (Figure 4c), we define a T-L hybrid plasmonic coupling mode based on the near-field coupling between the transversal mode and the longitudinal mode in the gold nanotube array, where the electric fields of the transversal and the longitudinal resonances present an overlay phenomenon. This T-L hybrid plasmonic coupling mode can be further clearly observed when changing the wall thickness of the gold nanotubes in the following sections.

Furthermore, the longitudinal resonance modes remarkably depend on the nanotube length. For instance, the longitudinal resonance mode L1 of the gold nanotubes with the length of 205 $\mathrm{nm}$ appear at $700 \mathrm{~nm}$ (Figure 4a) and redshifts to $870 \mathrm{~nm}$ when the nanotube length increases to $300 \mathrm{~nm}$ (Supporting Information Figure S2). It has been noted that there are some differences in the simulated and experimental peak positions, which may result from the fact that the gold nanotubes are assumed to have perfectly smooth surfaces in the simulations. In fact, the surfaces of the fabricated gold nanotubes are not very smooth, and the nanotube lengths are not very uniform, so some scattering losses at the surfaces of the nanorods are inevitable. On the other hand, during our FDTD simulation, to simplify the calculation, we select 1.61 as an effective refractive index of the AAO membranes. Actually, the refractive index depends on the wavelength, which presents a relatively large fluctuation when the wavelength is smaller than $1000 \mathrm{~nm}$ while that exhibits flat spectra when the wavelength is larger than $1000 \mathrm{~nm}$. In this case, the selected effective refractive index is close to its actual value at longer wavelengths but that deviates from the actual value at short wavelengths, which may result in the differences in the theoretical and experimental spectra at shorter wavelengths compared with those at longer wavelengths. Furthermore, the electric field distributions of the longitudinal resonance modes indicate the standing-wave modes present a three-quarter wave for both the $205 \mathrm{~nm}$ and $300 \mathrm{~nm}$ length nanotubes (Figure 4d and Figure S2 4d). The electric fields of the T-L hybrid plasmonic mode based on the coupling of the 


\section{WILEY-VCH}

transversal and the longitudinal resonances are still rather strong for the $300 \mathrm{~nm}$ length nanotube array (Figure S2 4c). However, the intensity of the electric fields of the longitudinal resonance mode L1 localized in the pores for the $300 \mathrm{~nm}$ length gold nanotubes (Figure S2 4d) remarkably reduces compared to those for the $205 \mathrm{~nm}$ length gold nanotubes, this is because the optical transmission coupling effect between the top and the bottom terminations of the gold nanotubes via the hollow nanochannels (i.e. pores) become weak when the nanotube length increase to $300 \mathrm{~nm}$. In contrast, when the nanotube length is up to $390 \mathrm{~nm}$, the T-L hybrid plasmonic mode based upon the coupling of the transversal and the longitudinal resonances is obviously asymmetrical and the electric fields of the hybrid mode at the incident ends (e.g., at the bottom of the nanotubes) are stronger than those at the emergent ends (e.g., at the top of the nanotubes) of the nanotube array (Figure $4 \mathrm{~g}$ ). This is because the coupling between the transversal and the longitudinal resonances at the incident ends is much stronger than that at the emergent ends since the electric fields of the transversal resonance mode are basically focused on the incident ends. Moreover, two longitudinal resonance modes L1 and L2 are found (Figure 4e), which are consistent with the experimental results, and the longitudinal resonance mode L1 presents a redshift from $1090 \mathrm{~nm}$ to $1204 \mathrm{~nm}$ and the longitudinal resonance mode L2 exhibits a redshift from $735 \mathrm{~nm}$ to $785 \mathrm{~nm}$ when the nanotube length increase from $390 \mathrm{~nm}$ to $440 \mathrm{~nm}$ (Figure 4e and Supporting Information Figure S3 a). It is interesting to note that the two longitudinal resonance modes L1 and L2 have different standing-wave modes, L1 shows a three-quarter standing-wave (Figure 4i and Figure S3 e) while L2 demonstrates a five-quarter standing-wave (Figure 4 h and Figure S3 d). In addition, both the three-quarter standing-wave and the five-quarter standing-wave modes are confined by a node at the incident ends with the weakest fields and an antinode at the emergent ends with the strongest fields (Figure $4 \mathrm{i}$ and h, Figure S3 d and e). The standingwave modes have the dispersion relation 


\section{WILEY-VCH}

$$
k_{i}=\frac{(2 i+1) \pi}{2 l}
$$

Where $k_{i}$ and $l$ are the wavenumber and the cavity length of the resonator (e.g., the nanotube length), respectively, $2 i+1$ is the order of the harmonics of the standing wave corresponding to quarter-wave modes. The resonance mode marked with L1 exhibits the same electric field distribution on the surface of the nanotubes with the different lengths from $205 \mathrm{~nm}$ to $440 \mathrm{~nm}$ (Figure $4 \mathrm{~d}$ and i, Figure $\mathrm{S} 2 \mathrm{~d}$ and Figure $\mathrm{S} \mathrm{3e}$ ), where $2 i+1=3(i=1)$ denotes the third order harmonic, and the standing-wave covers three quarter-wavelengths in the cavity. The resonance mode marked with L2 also demonstrates the same electric field distribution on the surface of the nanotubes with the different lengths of from $390 \mathrm{~nm}$ to $440 \mathrm{~nm}$ (Figure $4 \mathrm{~h}$ and Figure $S 3 \mathrm{~d})$, where $2 i+1=5(i=2)$ represents the fifth order harmonic, and the standing-wave contains five quarter-wavelengths in the cavity. Therefore, the harmonics of the standing waves with odd orders appears for the nanotubes with the length is less than $440 \mathrm{~nm}$. In addition, it is found that the electric fields of the longitudinal mode located in the pores of the nanotubes for the higher order harmonic (e.g., the fifth order mode L2) (Figure 4h and Figure S 3d) are stronger than those for the lower order harmonic (e.g., the third order mode L1, the electric field almost disappears) (Figure $4 \mathrm{i}$ and Figure $\mathrm{S} \mathrm{3e}$ ), which is resulted from the different intensity of the Fabry-Pérot resonances between the top and the bottom terminations of the metal nanotubes. Also, the electric fields of the longitudinal mode distributed in the pores of the nanotubes for the fifth order harmonic (mode L2) become weak with increasing the nanotube length from $390 \mathrm{~nm}$ to $440 \mathrm{~nm}$. Similarly, those electric fields for the third order harmonic (mode L1) exhibit the changes from rather strong, weak and then to disappeared when the nanotube length increases from $205 \mathrm{~nm}$ to $440 \mathrm{~nm}$. These results are attributed to the different coupling intensity of the standing-wave modes through the hollow nanochannels between the incident ends and the emergent ends of the nanotube arrays for the different lengths of the nanotubes. 


\section{WILEY-VCH}

It has been noted that, when the nanotube length increases to $670 \mathrm{~nm}$, the T-L hybrid plasmonic mode is not very obvious and its electric fields are mostly concentrated on the region close to the bottom sections covering the nanotube length of about $300 \mathrm{~nm}$ from the bottom section, which is associated with the weak coupling effect between the transversal and the longitudinal resonances owing to the long nanotubes (Figure 5c). Furthermore, the electric field distribution indicates the longitudinal resonance modes marked with L2, L3, and L4 performs a four-, six-, and eight-quarter waves or a two-, tree-, and four-half waves, respectively (Figure 5f, 5e and 5d), note that resonance mode L1 is out of the spectrum range. The standing-wave mode is confined by an antinode at both the incident and emergent ends, and the dispersion relation take the form

$$
k_{i}=\frac{2 i \pi}{2 l}
$$

Where the order of the harmonic is $2 i$, the resonance mode marked with L2 performs the standing-wave with four quarter-wavelengths in the cavity where $2 i=4(i=2)$ denotes the fourth harmonic, that marked with L3 demonstrates the standing-wave mode including six quarter-wavelengths where $2 i=6(i=3)$ represents the sixth order harmonic, that marked with L4 presents the standing-wave mode with eight quarter-wavelengths where $2 i=8(i=4)$ is the eighth order harmonic. Therefore, the higher-order harmonic modes in the nanotube resonators appear at the higher frequencies or shorter wavelengths, and the harmonics of the standing waves with even orders appears for the nanotubes when the nanotube length increases to $670 \mathrm{~nm}$. Similar to the longitudinal resonance modes with the odd order harmonics in the nanotube arrays with $390 \mathrm{~nm} 440 \mathrm{~nm}$ lengths, the electric fields of the longitudinal mode located in the pores of the nanotubes attenuate to zero with decreasing the order of the harmonic from the eighth, the sixth to the fourth orders.

In addition, it is observed that the thickness of the nanotube walls has remarkable effects on both the resonance wavelengths and the electric field distributions of the T-L hybrid 


\section{WILEY-VCH}

plasmonic mode and the longitudinal resonance mode. The resonance wavelength of the mode T1 sharply redshifts from $640 \mathrm{~nm}, 710 \mathrm{~nm}$ to $901 \mathrm{~nm}$ when the thickness of the nanotube walls reduces from $17 \mathrm{~nm}, 10 \mathrm{~nm}$ to $5 \mathrm{~nm}$ (Figure 6 a1-a3). It is found that the mode $\mathrm{T} 1$ is a typical T-L hybrid plasmonic mode based on the coupling between the longitudinal resonance mode with the seventh order harmonic $2 i+1=7 \quad(i=3)$ and the transversal resonance mode (Figure 6 b1-b3). When the walls of the gold nanotubes become very thin (e.g., $5 \mathrm{~nm}$ ), the electric field overlapping between the seventh order harmonic mode and the transversal resonance is much clear because of the strong coupling between the top and the bottom terminations of the gold nanotubes through the nanochannels based on the Fabry-Pérot resonances (Figure $6 \mathrm{~b} 3$ ), as a result the electric fields located in the nanochannels (pores) of the nanotubes at the incident ends are very strong and the intensity of the coupling fields exhibits a sharp decrease from the incident ends (bottom sections) to the emergent ends (top sections), which performs obvious asymmetry since the electric fields of the transversal resonance mode focus on the incident ends of the nanotube array. Moreover, the resonance wavelength of the mode L2 present remarkable redshifts from $786 \mathrm{~nm}, 836 \mathrm{~nm}$ to $1006 \mathrm{~nm}$ when the thickness of the nanotube walls reduces from $17 \mathrm{~nm}, 10 \mathrm{~nm}$ to $5 \mathrm{~nm}$ (Figure 6 a1a3). It is interesting to note that the mode L2 demonstrates a complex evolution from a typical longitudinal resonance mode with the fifth order harmonic (Figure $6 \mathrm{c} 1$ ) to the typically asymmetrical T-L hybrid plasmonic mode (Figure $6 \mathrm{c} 3$ ) with decreasing the wall thickness from $17 \mathrm{~nm}$ to $5 \mathrm{~nm}$. It is interesting to observe a middle configuration between the longitudinal mode and the asymmetrical T-L hybrid mode for the $10 \mathrm{~nm}$ thick walls (Figure 6 c2), the strong electric fields appears on both the incident ends and the emergent ends due to the strong Fabry-Pérot resonance coupling between the two ends via the nanochannels of the nanotubes, which performs an enhanced plasmon transmission phenomenon, where the hollow structures (i.e. pores) of the gold nanotubes work as a plasmonic channel of energy coupling. The enhanced plasmon transmission phenomenon can also be clearly observed in 


\section{WILEY-VCH}

the T-L hybrid plasmonic mode for the very thin walls of $5 \mathrm{~nm}$ in thickness. In this case, the mode L2 presents very strong asymmetrical T-L hybrid plasmonic mode in the gold nanotube array with the wall thickness of $5 \mathrm{~nm}$ (Figure $6 \mathrm{c} 3$ ), that is, the T-L hybrid mode based on the plasmonic coupling between the fifth order harmonic and the transversal mode is stronger than that based on the coupling between the seventh order harmonic and the transversal mode (Figure $6 \mathrm{c} 3$ and b3). Additionally, the electric fields of the longitudinal mode with the third order harmonic located in the pores of the nanotubes almost attenuate to zero for the $17 \mathrm{~nm}$ and $10 \mathrm{~nm}$ thick wall of the nanotubes (Figure $6 \mathrm{~d} 1$ and $6 \mathrm{~d} 2$ ). Nevertheless, the electric fields of the longitudinal mode with the third order harmonic are still rather strong for the very thin walls owing to the strong plasmonic coupling between the two ends through the nanochannels with large hollow space (Figure $6 \mathrm{~d} 3$ ).

\section{Conclusion}

We have studied, both theoretically and experimentally, novel plasmonic cavity resonators based on a metamaterial consisting of arrays of gold nanotubes embedded in anodic aluminum oxide membranes. Optical measurements have suggested that the longitudinal modes with different orders are excited when the nanotube length increases to specific values. The number of the high-order modes increases with the nanotube length, and the resonance mode shifts to higher frequencies or short wavelengths when the mode order grows. The longitudinal resonant modes of the same order red shift with increasing the length. Additionally, the resonant modes and electric field distributions in the plasmonic resonators based on the arrays of gold nanotubes have been simulated numerically by the FDTD method, the numerical results have been found to be consistent with the experimental data. Furthermore, the electric fields of the transversal resonance mode are concentrated at the incident edges of the nanotubes, but the electric fields of the longitudinal resonance modes are mainly localized at the outer surfaces of the nanotubes being partially distributed at the 


\section{WILEY-VCH}

interfaces between the air (pores) and inner walls as well as the two ends of the nanotubes, especially for the gold nanotube arrays with short lengths, thin walls and higher order harmonics. In addition, the electric fields on the surfaces of the gold nanotube walls have standing-wave patterns for the longitudinal resonance modes with different orders, therefore, the arrays of gold nanotubes can serve as efficient plasmonic resonators, which originates from the Fabry-Pérot resonances between the metal terminations. Moreover, the standing waves with odd and even orders appear for the different nanotube lengths, and they can be controlled by the nanotube length. On the other hand, we have found the T-L hybrid plasmonic modes with asymmetrical distribution of the electric field, which originates from the plasmonic near-field coupling between the transversal and longitudinal modes with the different orders. We have demonstrated that the thickness of the nanotube walls has a remarkable effect on the T-L hybrid plasmonic modes, and the electric fields of the T-L hybrid plasmonic mode is enhanced significantly with decreasing the nanotube walls, in particular, the longitudinal resonance modes with both seventh and fifth orders couple to the transversal resonance modes for very thin walls of $5 \mathrm{~nm}$ and excite the T-L hybrid modes. We have also found that the electric fields of the longitudinal mode localized in the pores of the nanotubes depend strongly on the harmonic order of the standing-wave modes and the lengths as well as the nanotube walls. We believe that these novel plasmonic resonant cavities based on the arrays of gold nanotubes can provide the basis for applications in nanophotonic devices.

\section{Experimental Section}

Synthesis of anodic aluminum oxide (AAO) membranes: First, AAO membranes were fabricated by a two-step anodization method. High-purity aluminum sheets $(99.999 \%, 0.3 \mathrm{~mm}$ thick) were degreased in acetone, and then an annealing treatment under vacuum $\left(10^{-4} \mathrm{~Pa}\right)$ was carried out at $500{ }^{\circ} \mathrm{C}$ for $5 \mathrm{~h}$ to remove the mechanical stress and make the grains grow up. The aluminum sheets were electropolished at $23 \mathrm{~V}$ in a 1:9 mixture solution of perchloric 


\section{WILEY-VCH}

acid and ethanol at $2{ }^{\circ} \mathrm{C}$. Then, the aluminum sheets were anodized under a constant voltage of $40 \mathrm{~V}$ in a $0.3 \mathrm{M}$ oxalic acid solution at $5{ }^{\circ} \mathrm{C}$ for $10 \mathrm{~h}$. After that, the anodic oxide layers formed on the sheets were removed in a mixture solution of $6 \% \mathrm{H}_{3} \mathrm{PO}_{4}$ and $1.8 \% \mathrm{H}_{2} \mathrm{CrO}_{4}$ at $60{ }^{\circ} \mathrm{C}$. Subsequently, those sheets were correspondingly anodized again under the constant voltage of $40 \mathrm{~V}$ in the $0.3 \mathrm{M}$ oxalic acid solution at $5{ }^{\circ} \mathrm{C}$ for $10 \mathrm{~h}$. After, the aluminum substrates were removed in a $\mathrm{SnCl}_{4}$ solution, the through-channel AAO membranes were achieved by a chemical etching treatment in a $5 \% \mathrm{H}_{3} \mathrm{PO}_{4}$ solution at $30{ }^{\circ} \mathrm{C}$. Finally, the nanochannel sizes of the AAO membranes were tuned by control of the etching duration.

Preparation of gold nanotube arrays: The gold nanotube arrays were synthesized by using a galvanostatic electrodeposition process into the nanochannels of AAO (Figure $1 \mathrm{a}(1)$ ) in a conventional three-electrode cell (Supporting Information Figure S4). A very thin layer of gold film $(\sim 50 \mathrm{~nm})$ with a tube-like framework geometry sputtered onto the surfaces of the AAO membranes was served as a working electrode (Figure $1 \mathrm{a}(2)$ ), where the framework geometry was worked as nucleation points to grow the gold nanotubes, the graphite and the $\mathrm{Ag} / \mathrm{AgCl}$ electrode were used as a counter and a reference electrode, respectively. The experiment was performed in the three-electrode cell by an electrochemical workstation (VersaSTAT 4, Princeton Applied Research (PAR)) and VersaStudio control software. An aqueous solution containing $\mathrm{HAuCl}_{4}(5 \mathrm{~g} / \mathrm{L})$, ethylene diamine tetraacetic acid $(2.5 \mathrm{~g} / \mathrm{L})$, $\mathrm{Na}_{2} \mathrm{SO}_{3}(80 \mathrm{~g} / \mathrm{L})$, and $\mathrm{K}_{2} \mathrm{HPO}_{4}(15 \mathrm{~g} / \mathrm{L})$ was used to prepare gold nanotube arrays. Prior to the electrochemical deposition, the AAO membranes were immersed in the electrolyte solution under for 2 min to ensure wetting of the pores of the AAO membranes. The Au nanotubes were electrodeposited into the pores (Figure $1 \mathrm{a}(3)$ ) at a constant current of $-0.5 \mathrm{~mA}$ (vs $\mathrm{Ag} / \mathrm{AgCl}$ ) by a chronopotentiometry technique action in the electrochemical workstation. The outer diameter of the gold nanotubes was controlled by the channel diameters of the AAO membranes, and the inner diameter of the gold nanotubes was tuned by the thickness of the 


\section{WILEY-VCH}

$\mathrm{Au}$ film electrode. The length of the gold nanotube arrays was adjusted by the electrodeposition time from 500 s, 600 s, 900 s, 1600 s, and 1900 s. Finally, the Au film as the electrode layer was removed carefully from the bottom surfaces of the membranes by a fine polishing method (Figure $1 \mathrm{a}(4)$ ), and the gold nanotube arrays embedded in AAO membranes with transparent deep red color were obtained. Finally, the Au nanotube arrays embedded in the AAO membranes were characterized by field-emission scanning electron microscopy (FE-SEM, Sirion 200).

Measurements of reflection spectra of gold nanotube arrays: Reflectance measurements of the Au nanotube arrays embedded in AAO membranes were performed on a UV-Vis-NIR optical spectrometer (Shimadzu UV3600) with a fixed-angle reflector $\left(8^{\circ}\right)$ and calibrated against a diffuse reflectance module.

FDTD Simulation Section: Finite difference time domain (FDTD) simulations were conducted with lumerical solutions (commercial software, Canada). The calculation was performed in a space of $1150 \mathrm{~nm} \times 1150 \mathrm{~nm} \times 1000 \mathrm{~nm}$ under perfectly matched layer boundary conditions in $\mathrm{x}, \mathrm{y}$ and $\mathrm{z}$ directions. The background index of the simulation volume was 1.61 . We used the Drude model to determine the dielectric function of gold and the data base of the permittivity was given by Johnson Christy. ${ }^{[52]}$ A broadband TE-polarized plane wave (300 nm to $2200 \mathrm{~nm}$ ) with size of $1050 \mathrm{~nm} \times 1050 \mathrm{~nm}$ in a $\mathrm{x}-\mathrm{y}$ plane, underneath the bottom of the whole hexagonal packing gold nanotubes array, was employed to propagate in the positive zdirection with a specific inclined angle ( $8^{\circ}$ deviation from the $\mathrm{z}$-axis), which is consistent with the experimental configuration. The mesh size was high accuracy auto non-uniform type to ensure its sufficiency for simulation meanwhile with an appropriate computer memory costs. ${ }^{[53]}$ The simulated reflectance spectra were obtained from the $\mathrm{x}-\mathrm{y}$ plane below the light source plane with a distance of $150 \mathrm{~nm}$. The field distributions of hybrid nanocavity array were extracted from a $650 \mathrm{~nm} \times 600 \mathrm{~nm}$-z plane located in the middle of nanotube arrays. 


\section{Supporting Information}

Supporting Information is available from the Wiley Online Library or from the author.

\section{Acknowledgements}

This work was supported by the Seventh Framework Program of the European Union for Research through Marie Curie International Incoming Fellowships (FP7-PEOPLE-2013-IIF, Grant Nos. 623473, 913473), National Basic Research Program of China (Grant No. 2012CB932303, 2012CB921900), National Natural Science Foundation of China (Grant No. 10804112), the Australian Research Council, and the Leverhulme Trust through the Visiting Professorship. Author 1 (Junfeng Wang) and Author 2 (Cheng Zhang) contributed equally to this work.

Received: ((will be filled in by the editorial staff))

Revised: ((will be filled in by the editorial staff)) Published online: ((will be filled in by the editorial staff))

[1] N. P. de Leon, B. J. Shields, C. L. Yu, D. E. Englund, A. V. Akimov, M. D. Lukin, H. Park, Phys. Rev. Lett. 2012, 108, 226803.

[2] P. Ginzburg, Rev. Phys. 2016, 1, 120.

[3] E. M. Purcell, Phys. Rev. 1946, 69, 681.

[4] K. J. Vahala, Nature 2003, 424, 839.

[5] P. Muhlschlegel, H. J. Eisler, O. J. F. Martin, B. Hecht, D. W. Pohl, Science 2005, 308, 1607.

[6] C. E. Hofmann, F. J. G. de Abajo, H. A. Atwater, Nano Lett. 2011, 11, 372.

[7] L. Novotny, N. van Hulst, Nat. Photon. 2011, 5, 83.

[8] E. Palacios, A. Chen, J. Foley, S. K. Gray, U. Welp, D. Rosenmann, V. K. Vlasko-Vlasov, Adv. Opt. Mater. 2014, 2, 394. 


\section{WILEY-VCH}

[9] R. Chikkaraddy, B. de Nijs, F. Benz, S. J. Barrow, O. A. Scherman, E. Rosta, A. Demetriadou, P. Fox, O. Hess, J. J. Baumberg, Nature 2016, 535, 127.

[10] A.V. Akimov, A. Mukherjee, C. L. Yu, D. E. Chang, A. S. Zibrov, P. R. Hemmer, H. Park, M. D. Lukin, Nature 2007, 450, 402.

[11] R. F. Oulton, V. J. Sorger, T. Zentgraf, R. M. Ma, C. Gladden, L. Dai, G. Bartal, X. Zhang, Nature 2009, 461, 629.

[12] V. J. Sorger, Z. Ye, R. F. Oulton, Y. Wang, G. Bartal, X. Yin, X. Zhang, Nat. Commun. 2011, 2, 331 .

[13] W. Zhou, M. Dridi, J. Y. Suh, C. H. Kim, D. T. Co, M. R. Wasielewski, G. C. Schatz, T. W. Odom, Nat. Nanotechnol. 2013, 8, 506.

[14] C. Xiang, C. K. Chan, J. Wang, Sci. Rep. 2014, 4, 3720.

[15] C. Sauvan, J. P. Hugonin, I. S. Maksymov, P. Lalanne, Phys. Rev. Lett. 2013, 110, 345.

[16] C. Zhang, Y. H. Lu, Y. Ni, M. Z. Li, L. Mao, C. Liu, D. G. Zhang, H. Ming, P. Wang, Nano Lett. 2015, 15, 1382.

[17] E. S. Barnard, J. S. White, A. Chandran, M. L. Brongersma, Opt. Express 2008, 16, 16529.

[18] P. Bharadwaj, B. Deutsch, L. Novotny, Adv. Opt. Photon. 2009, 1, 438.

[19] D. K. Gramotnev, A. Pors, M. Willatzen, S. I. Bozhevolnyi, Phys. Rev. B 2012, 85, 045434.

[20] A. V. Kabashin, P. Evans, S. Pastkovsky, W. Hendren, G. A. Wurtz, R. Atkinson, R. Pollard, V. A. Podolskiy, A. V. Zayats, Nat. Mater. 2009, 8, 867.

[21] M. Muradoglu, C. Y. Lau, I. Gralinski, T. W. Ng, Plasmonics 2014, 9, 367.

[22] K. Bhattarai, Z. Ku, S. Silva, J. Jeon, J. O. Kim, S. J. Lee, A. Urbas, J. Zhou, Adv. Opt. Mater. 2015, 3, 1779.

[23] V. Giorgis, P. Zilio, G. Ruffato, M. Massari, G. Zacco, F. Romanato, Opt. Express 2014, 22, 26476. 


\section{WILEY-VCH}

[24] J. A. Schuller, E. S. Barnard, W. Cai, Y. C. Jun, J. S. White, M. L. Brongersma, Nat. Mater. 2010, 9, 193.

[25] G. A. Wurtz, R. Pollard, W. Hendren, G. P. Wiederrecht, D. J. Gosztola, V. A. Podolskiy, A. V. Zayats, Nat. Nanotechnol. 2011, 6, 107.

[26] M. Kauranen, A. V. Zayats, Nat. Photon. 2012, 6, 737.

[27] J. Lee, N. Nookala, J. S. Gomez-Diaz, M. Tymchenko, F. Demmerle, G. Boehm, M.-C. Amann, A. Alu, M. A. Belkin, Adv. Opt. Mater. 2016, 4, 664.

[28] H. A. Atwater, A. Polman, Nat. Mater. 2010, 9, 205.

[29] M. A. Sefunc, A. K. Okyay, H. V. Demir, Appl. Phys. Lett. 2011, 98, 093117.

[30] H. Ditlbacher, A. Hohenau, D. Wagner, U. Kreibig, M. Rogers, F. Hofer, F. R. Aussenegg, J. R. Krenn, Phys. Rev. Lett. 2005, 95, 257403.

[31] N. P. de Leon, B. J. Shields, C. L. Yu, D. E. Englund, A. V. Akimov, M. D. Lukin, H. Park, Phys. Rev. Lett. 2012, 108, 226803.

[32] L. Novotny, Phys. Rev. Lett. 2007, 98, 266802.

[33] E. Cubukcu, F. Capasso, Appl. Phys. Lett. 2009, 95, 201101.

[34] S. I. Bozhevolnyi, T. Søndergaard, Opt. Express 2007, 15, 10869.

[35] G. D. Valle, T. Søndergaard, S. I. Bozhevolnyi, Opt. Express 2008, 16, 6867.

[36] J. Jung, T. Søndergaard, S. I. Bozhevolnyi, Phys. Rev. B 2009, 79, 035401.

[37] P. Berini, Adv. Opt. Photon. 2009, 1, 484.

[38] P. Berini, I. De Leon, Nat. Photon. 2011, 6, 16.

[39] J. X. Zhang, L. D. Zhang, Adv. Opt. Photon. 2012, 4, 157.

[40] G. D. Valle, T. Søndergaard, S. I. Bozhevolny, Opt. Express 2008, 16, 6867.

[41] T. Søndergaard, S. I. Bozhevolnyia, J. Junga, J. Beermannb, G. D. Valle, A. E. Boltasseva, Proc. of SPIE 2008, 7099, 70991G.

[42] A. Chandran, E. S. Barnard, J. S. White, M. L. Brongersma, Phys. Rev. B 2012, 85, 085416. 


\section{WILEY-VCH}

[43] T. Siegfried, Y. Ekinci, O. J. F. Martin, H. Sigg, Nano Lett. 2013, 13, 5449.

[44] S. A. Maier, Plasmonics: Fundamentals and Applications (Springer, 2007).

[45] D. P. Lyvers, J. M. Moon, A.V. Kildishev, V. M. Shalaev, A. Wei, ACS Nano 2008, 2, 2569.

[46] M. K. Gupta, T. Koenig, R. Near, D. Nepal, L. F. Drummy, S. Biswas, S. Naik, R. A. Vaia, M. A. El-Sayed, V. V. Tsukruk, Small 2013, 9, 2979.

[47] M. Bora, B. J. Fasenfest, E. M. Behymer, A. S-P Chang, H. T. Nguyen, J. A. Britten, C. C. Larson, J. W. Chan, R. R. Miles, T. C. Bond, Nano Lett. 2010, 10, 2832.

[48] T. W. Ebbesen, H. J. Lezec, H. F. Ghaemi, T. Thio, P. A. Wolff, Nature 1998, 391, 667.

[49] H. F. Ghaemi, T. Thio, D. E. Grupp, T. W. Ebbesen, H. J. Lezec, Phys. Rev. B 1998, 58, 6779.

[50] C. Genet, T. W. Ebbesen, Nature 2007, 445, 39.

[51] T. S. Eriksson, A. Hjortsberg, G. A. Niklasson, C. G. Granqvist, Appl. Opt. 1981, 20, 2742.

[52] P. B. Johnson, R. W. Christy, Phys. Rev. B 1972, 6, 4370.

[53] https://kb.lumerical.com/en/ref_sim_obj_non-uniform_mesh.html. 


\section{WILEY-VCH}

a

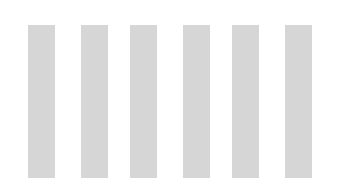

(1)

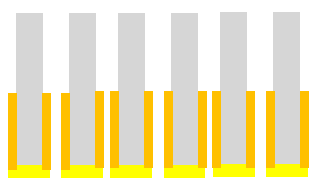

(3)
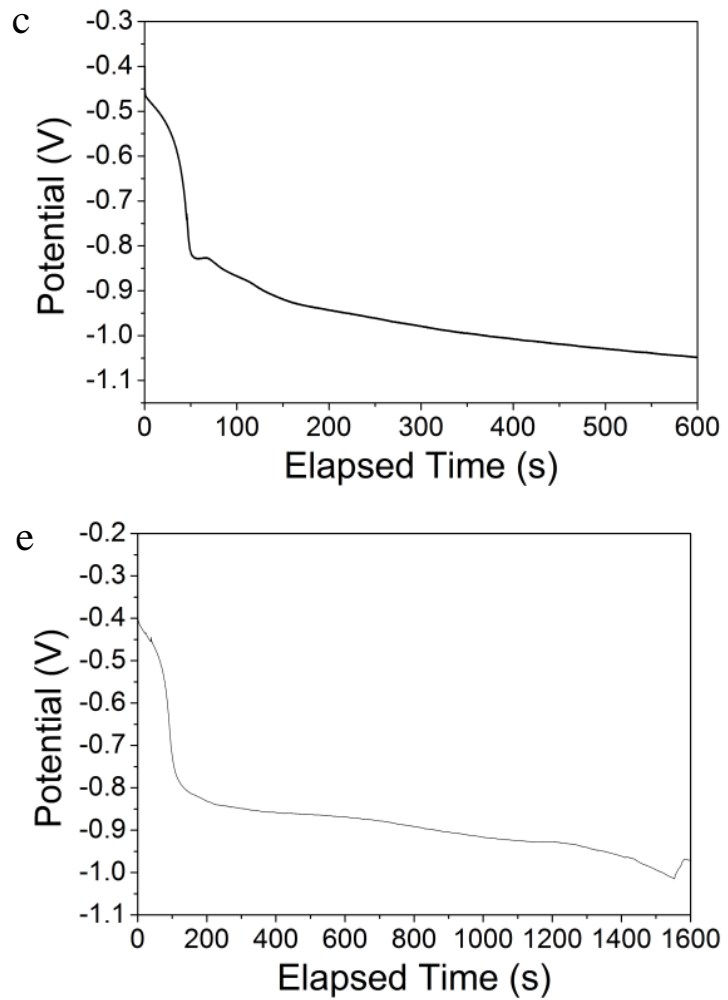

b
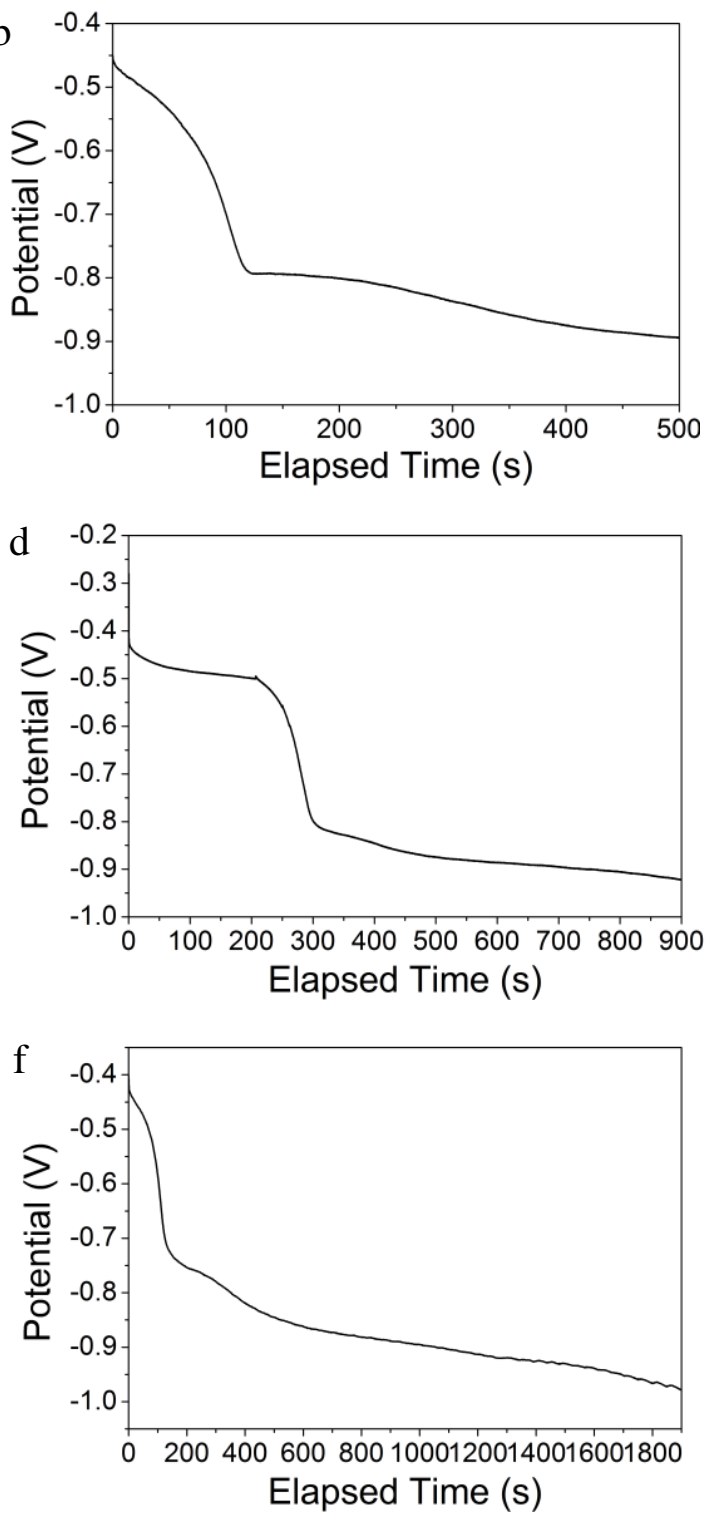

Figure 1. (a) Schematic illustrations of fabrication process of gold nanotubes by the AAO template based synthesis approach: (1) The AAO membrane; (2) Coating a very thin layer of gold film served as a working electrode on the bottom surface of the AAO membrane; (3) Electrodepositing Au nanotubes into the nanopores by a chronopotentiometry technique in the PAR electrochemical workstation; (4) Removal of the Au film electrode layer to achieve plasmonic gold nanotube arrays. (b-f) Deposition potential with elapsed time during the electrodeposition processes under a constant current of $-0.5 \mathrm{~mA}$ ( $\mathrm{vs} \mathrm{Ag} / \mathrm{AgCl}$ ), the length of the Au nanotubes was controlled by the electrodeposition time for $500 \mathrm{~s} \mathrm{(b),600} \mathrm{s} \mathrm{(c),} 900 \mathrm{~s}$ (d), $1600 \mathrm{~s} \mathrm{(e),} \mathrm{and} 1900 \mathrm{~s}$ (f). 


\section{WILEY-VCH}

a

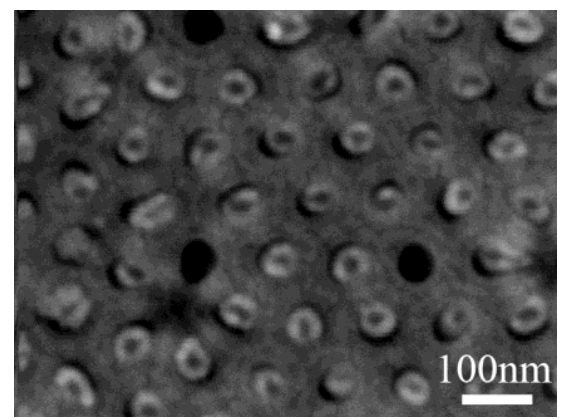

b

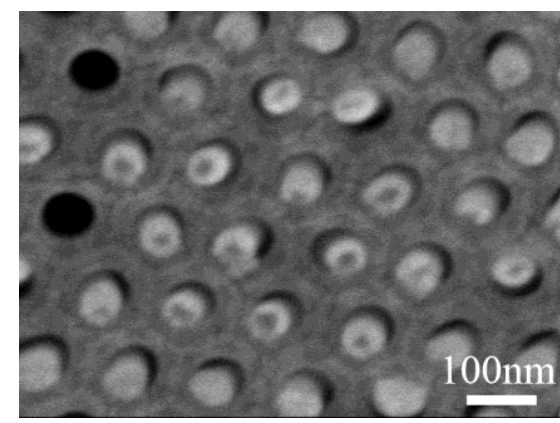

c

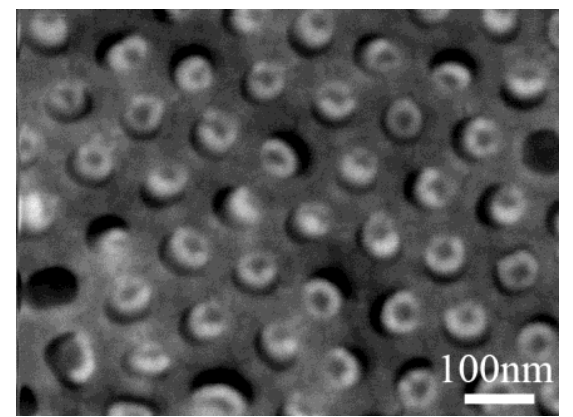

d

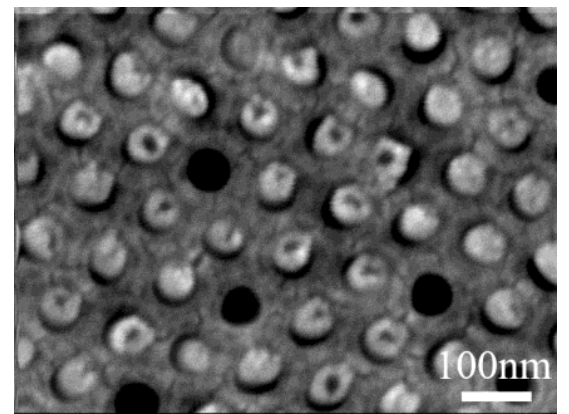

e

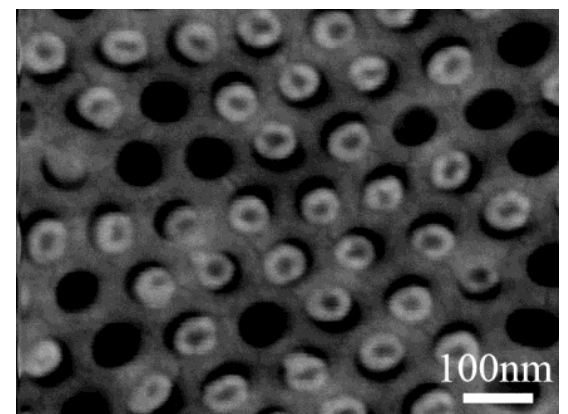

f

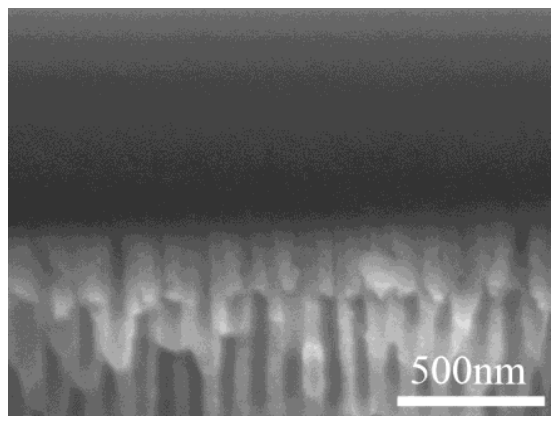

g

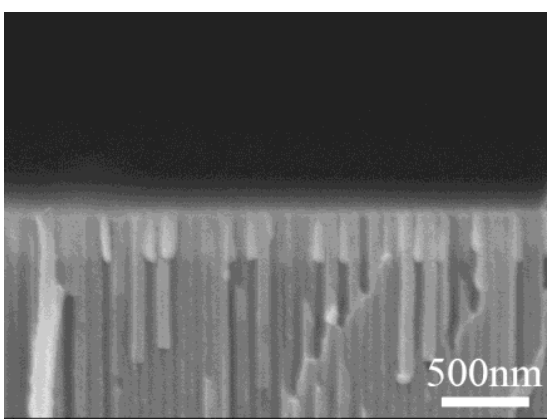

h

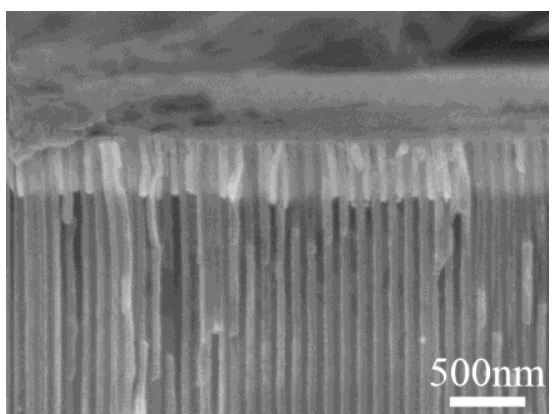

i

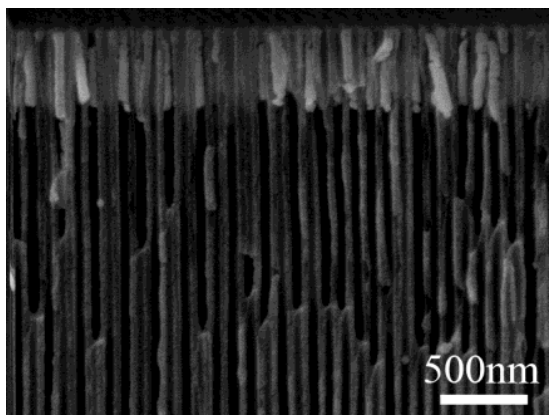

j

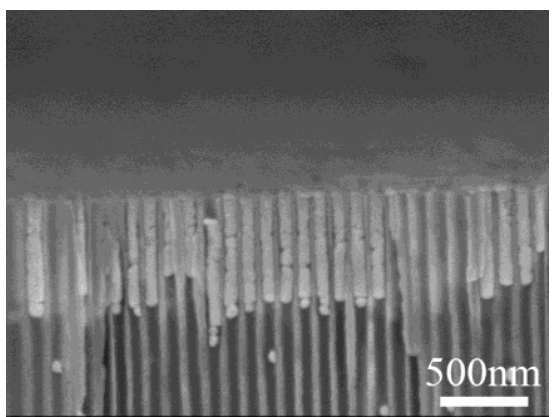

Figure 2. (a-e) Top views of SEM images of gold nanotube arrays embedded in AAO membranes with different lengths of $205 \pm 17 \mathrm{~nm}, 300 \pm 9 \mathrm{~nm}, 390 \pm 17 \mathrm{~nm}, 440 \pm 29 \mathrm{~nm}$ to $670 \pm 39 \mathrm{~nm}$, respectively. (f-j) Cross sectional views of SEM images of the corresponding gold nanotube arrays. 

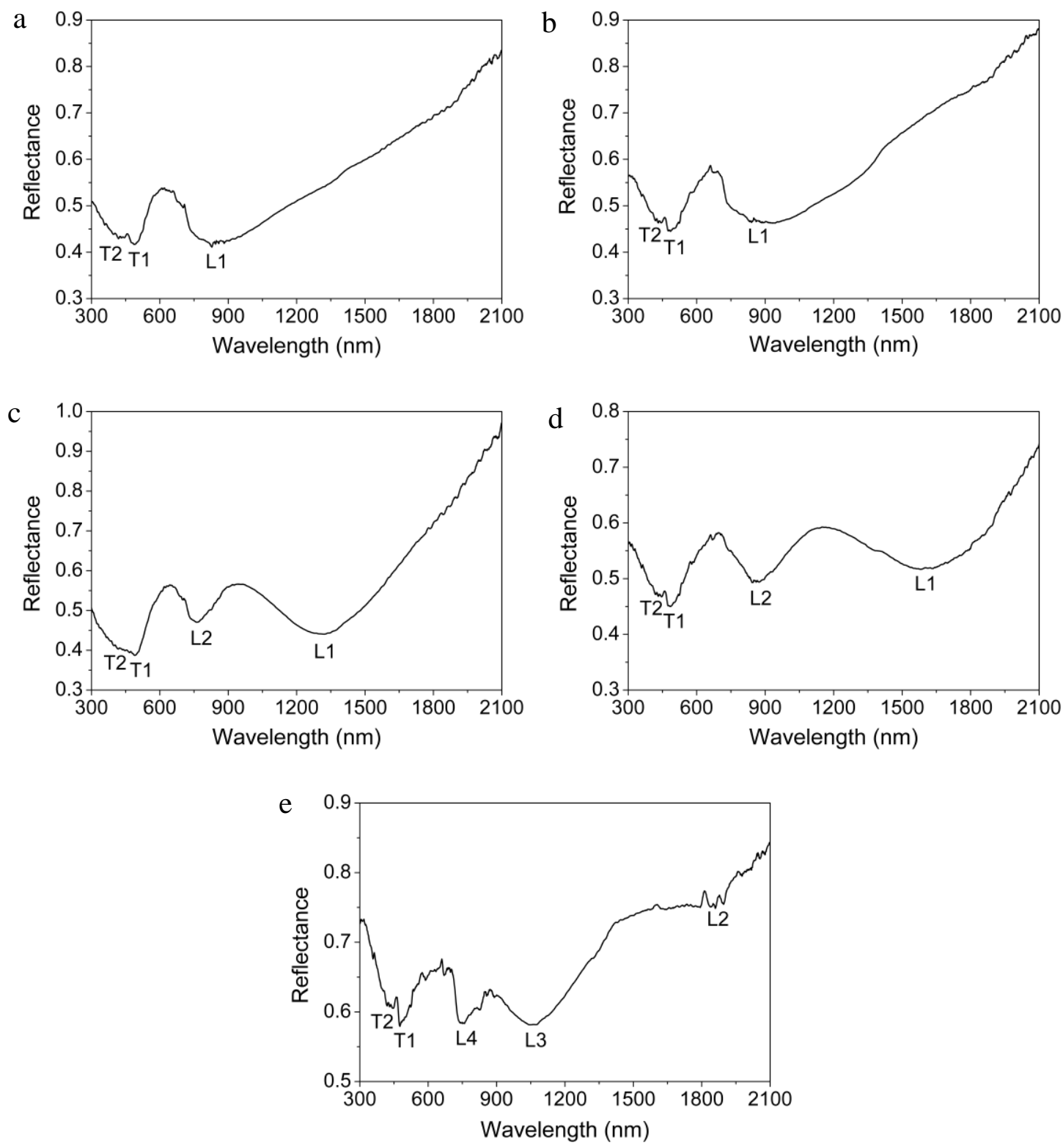

Figure 3. UV-Vis-NIR reflectance spectra of the gold nanotube arrays with the different lengths of $205 \mathrm{~nm}$ (a), $300 \mathrm{~nm}$ (b), $390 \mathrm{~nm}$ (c), $440 \mathrm{~nm}$ (d), and $670 \mathrm{~nm}$ (e), respectively. 


\section{WILEY-VCH}
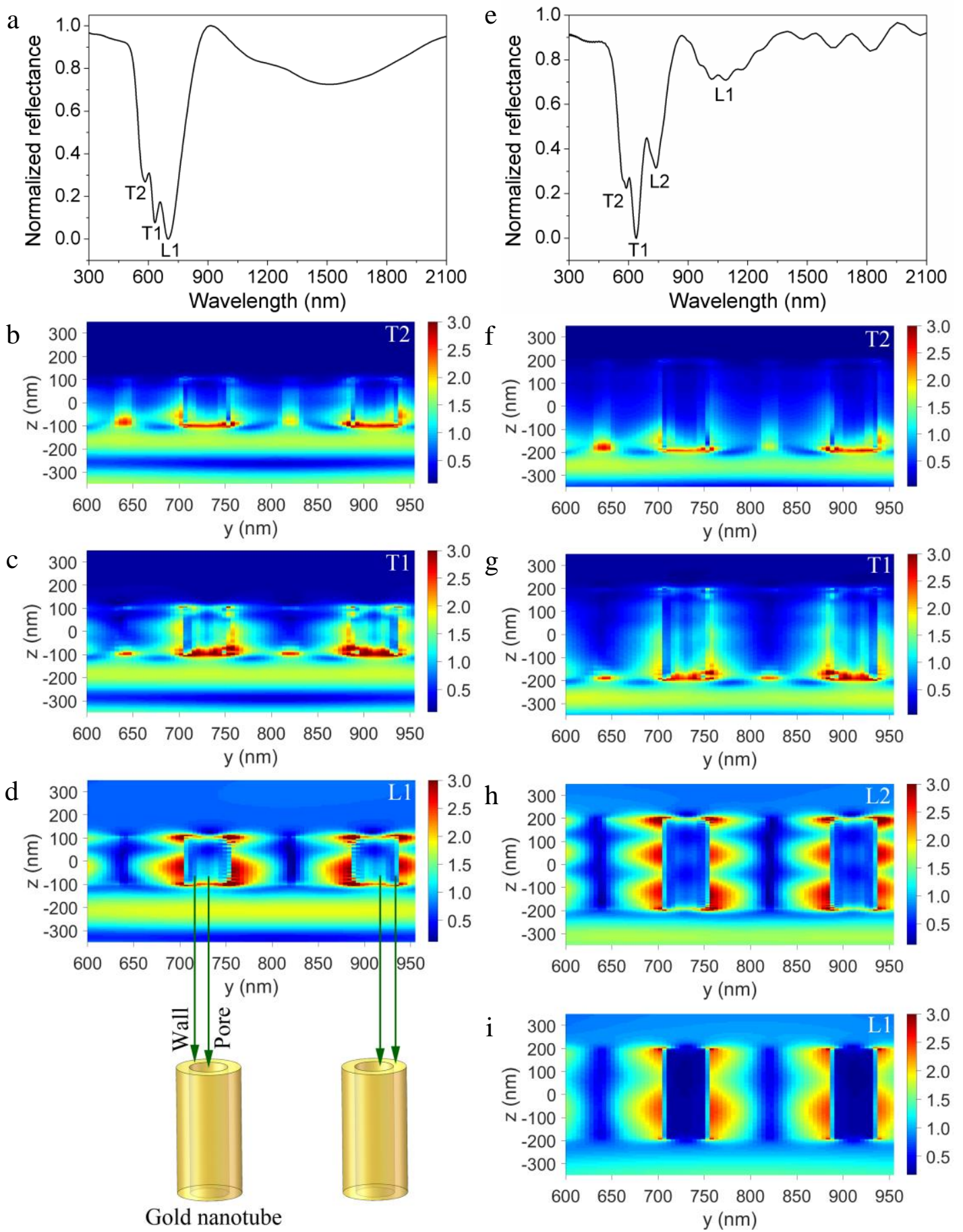

Figure 4. (a) Reflectance spectra of the gold nanotube array with the length of $205 \mathrm{~nm}$ by FDTD simulation, (b) Electric field distribution of the transverse resonance mode T2 for the nanotubes of $205 \mathrm{~nm}$ in length, (c) Electric field distribution of the hybrid plasmonic mode T1 of the $205 \mathrm{~nm}$ long nanotube array, (d) Electric field distribution of the longitudinal resonance mode L1, inset: schematic representation of the wall and the pore of the gold nanotube; (e) Reflectance spectra of the gold nanotube array with the length of $390 \mathrm{~nm}$ by FDTD simulation, (f) Electric field distribution of the transverse resonance mode T2 for the nanotubes of $390 \mathrm{~nm}$ in length, (g) Electric field distribution of the hybrid plasmonic mode T1 of the $390 \mathrm{~nm}$ long nanotube array, $(h, i)$ the electric field distributions of the longitudinal resonance modes L2 and L1 with the different orders of the harmonics, respectively. 
a

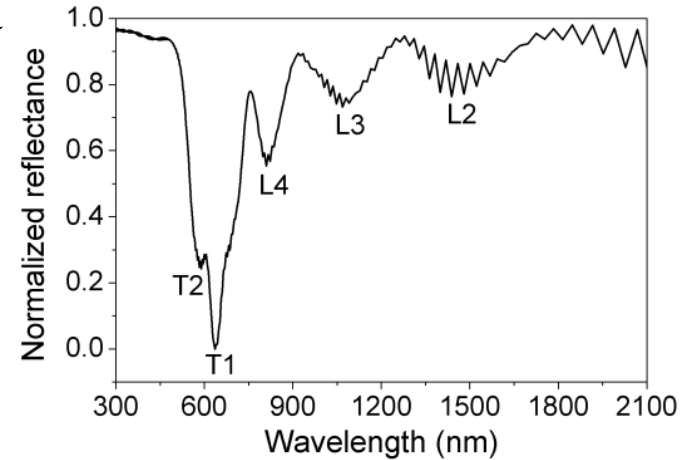

b
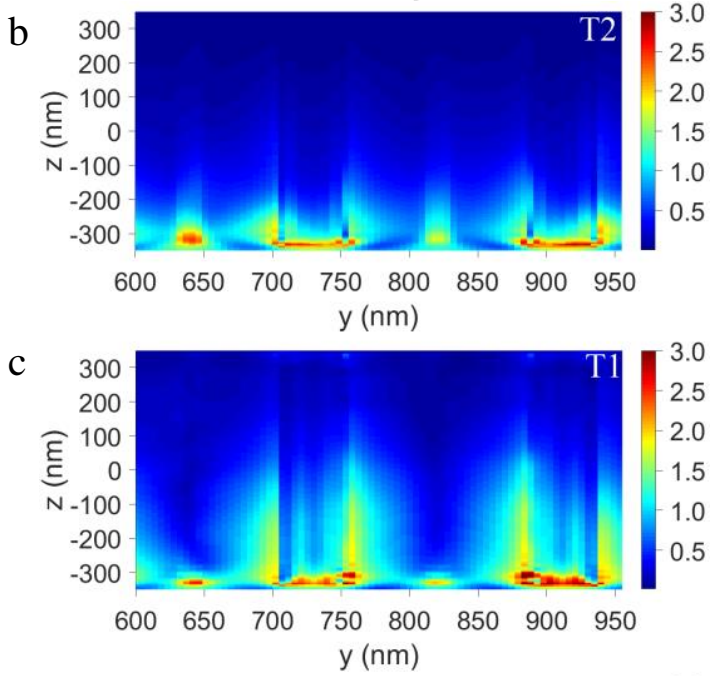

d

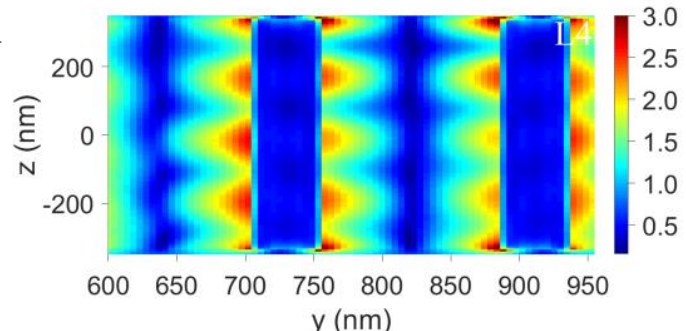

e
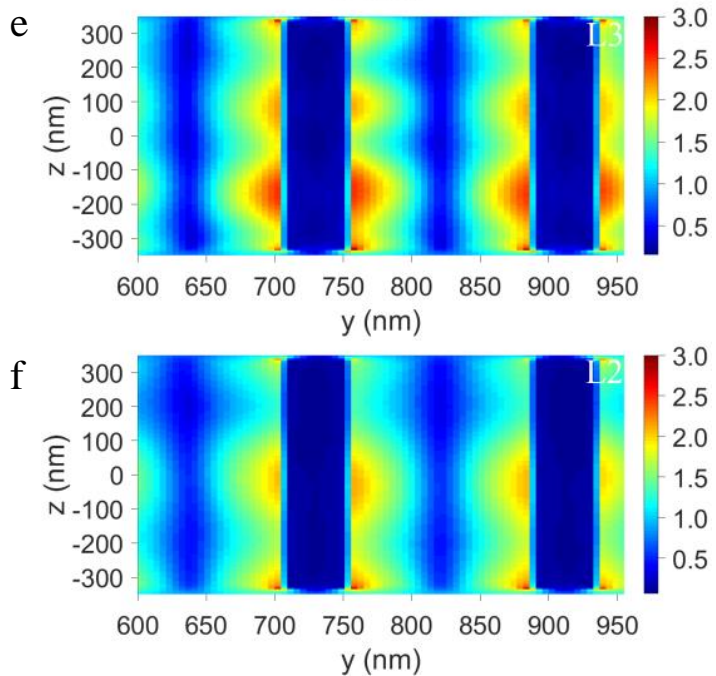

Figure 5. Reflectance spectra of the gold nanotube array with the length of $670 \mathrm{~nm}$ by FDTD simulation, Where, T2 denotes the transverse resonance mode, T1 represents the hybrid plasmonic mode, and L2, L3 and L4 represent the longitudinal resonance modes related to the different orders of harmonics. (b, c) Corresponding to the electric field distributions of the modes $\mathrm{T} 2$ and $\mathrm{T} 1,(\mathrm{~d}, \mathrm{e}, \mathrm{f})$ represent the electric field distributions of the 1 modes L4, L3 and L2 with different orders of harmonics, respectively. 


\section{WILEY-VCH}
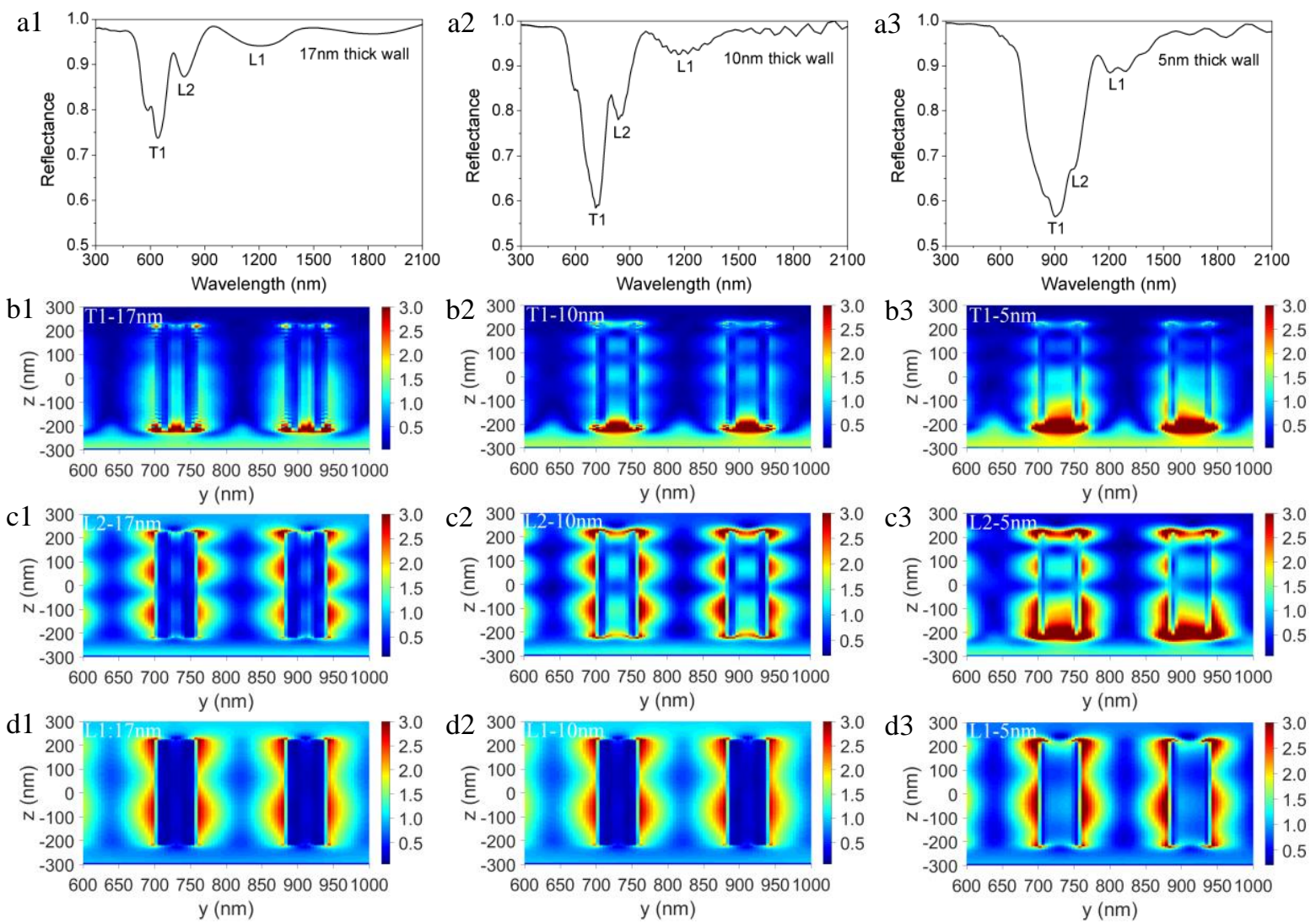

Figure 6. (a1, a2, a3) Reflectance spectra of the gold nanotube arrays with the different wall thicknesses of $17 \mathrm{~nm}, 10 \mathrm{~nm}$ and $5 \mathrm{~nm}$ when the length and the outer diameter of the nanotubes are fixed at $440 \mathrm{~nm}$ and $54 \mathrm{~nm}$, respectively. (b1, b2, b3) Correspondingly to the electric field distributions of the hybrid plasmonic mode T1 for the $17 \mathrm{~nm}, 10 \mathrm{~nm}$ and $5 \mathrm{~nm}$ thick nanotube walls. (c1, c2, b3) Electric field distributions of the longitudinal resonance modes L2 for the $17 \mathrm{~nm}, 10 \mathrm{~nm}$ and the hybrid plasmonic mode L2 for $5 \mathrm{~nm}$ thick nanotube walls, respectively. (d1, d2, d3) Electric field distributions of the longitudinal resonance mode $\mathrm{L} 1$ for the $17 \mathrm{~nm}, 10 \mathrm{~nm}$ and $5 \mathrm{~nm}$ thick nanotube walls, respectively. 


\section{WILEY-VCH}

Gold nanotube arrays worked as efficient plasmonic cavity resoantors are successfully synthesized by a template-based method. Odd and even harmonics with standing-wave modes are tuned by nanotube length. T-L hybrid cavity modes dependent on wall thickness and length of nanotubes, perform electric fields localized into pores and at two ends based on extraordinary optical transmission coupling effect through inner-nanochannels.

\section{Keyword}

plasmonic resonator, cavity mode, hybrid resonance mode, gold nanotube, array

Junfeng Wang, Cheng Zhang, Junxi Zhang*, Haojie Song, Pei Wang, Yonghua Lu, Guangtao Fei, Wen $\mathrm{Xu}$, Wei Xu, Lide Zhang, Yuri S. Kivshar, and Lin Zhang

\section{Hybrid plasmonic cavity modes in arrays of gold nanotubes}

\section{ToC figure}

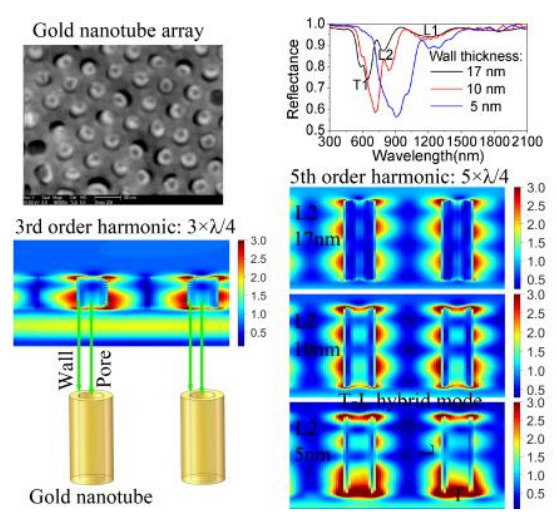




\section{WILEY-VCH}

Copyright WILEY-VCH Verlag GmbH \& Co. KGaA, 69469 Weinheim, Germany, 2013.

\section{Supporting Information}

\section{Hybrid plasmonic cavity modes in arrays of gold nanotubes}

Junfeng Wang, Cheng Zhang, Junxi Zhang*, Haojie Song, Pei Wang, Yonghua Lu, Guangtao Fei, Wen Xu, Wei Xu, Lide Zhang, Yuri S. Kivshar, and Lin Zhang

J. Wang, Dr. J. Zhang, H. Song, Dr. G. Fei, W(ei). Xu, Prof. L. D. Zhang

Key Laboratory of Materials Physics

Anhui Key Laboratory of Nanomaterials and Nanostructures

Institute of Solid State Physics

Chinese Academy of Sciences

Hefei 230031, P. R. China

E-mail: jxzhang@issp.ac.cn

C. Zhang, Prof. P. Wang, Dr. Y. Lu

Department of Optics and Optical Engineering

University of Science and Technology of China

Hefei 230026, P. R. China

Dr. J. Zhang, Prof. L. Zhang

Aston Institute of Photonic Technologies

School of Engineering \& Applied Science

Aston University

Birmingham B4 7ET, UK

Dr. W(en). Xu

Research Laboratory of Computational Materials Sciences

Institute of Solid State Physics

Chinese Academy of Sciences

Hefei 230031, P. R. China

Prof. Y. S. Kivshar

Nonlinear Physics Centre

Research School of Physics and Engineering

Australian National University

Canberra ACT 2601, Australia 
a

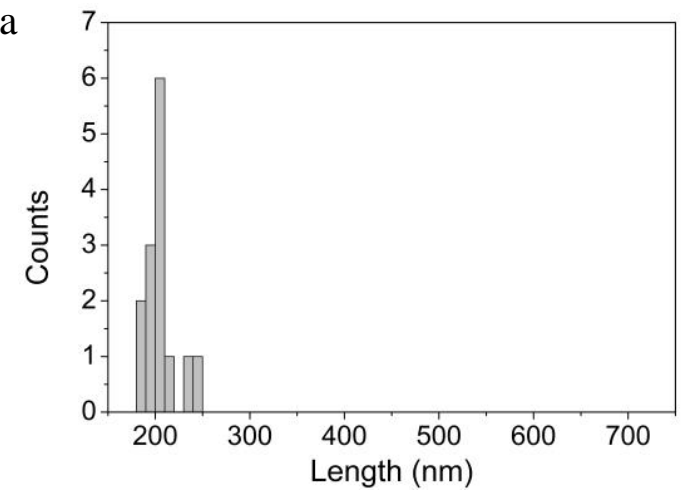

$\mathrm{c}$

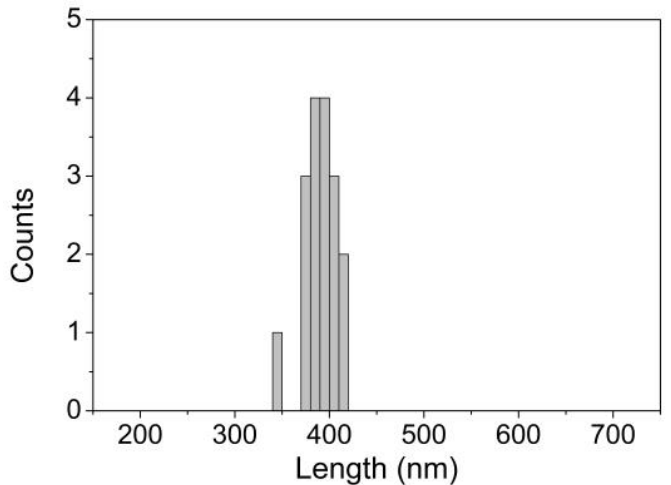

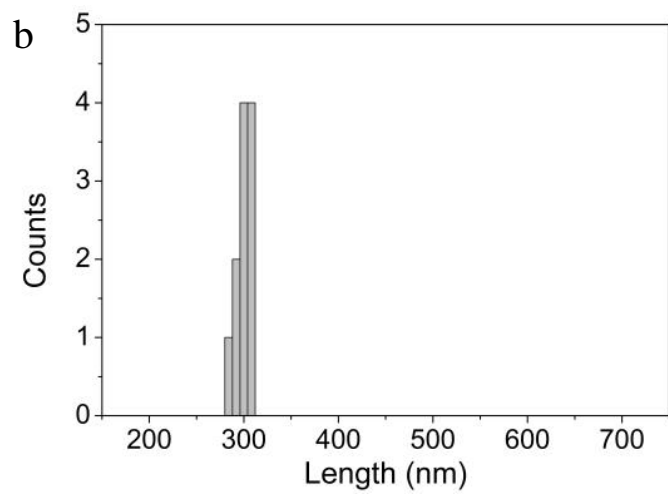

d

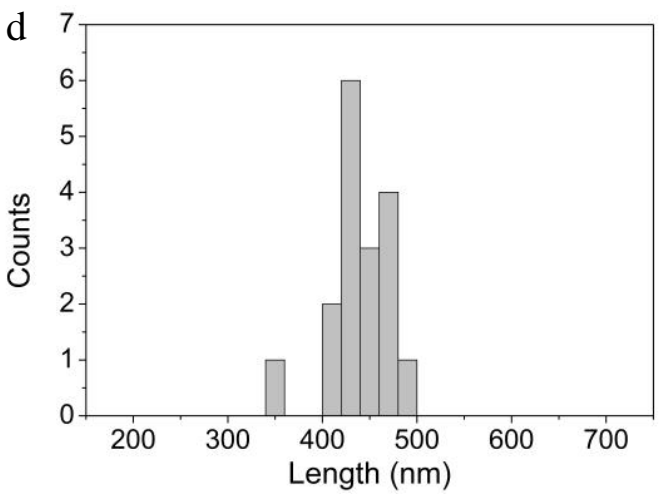

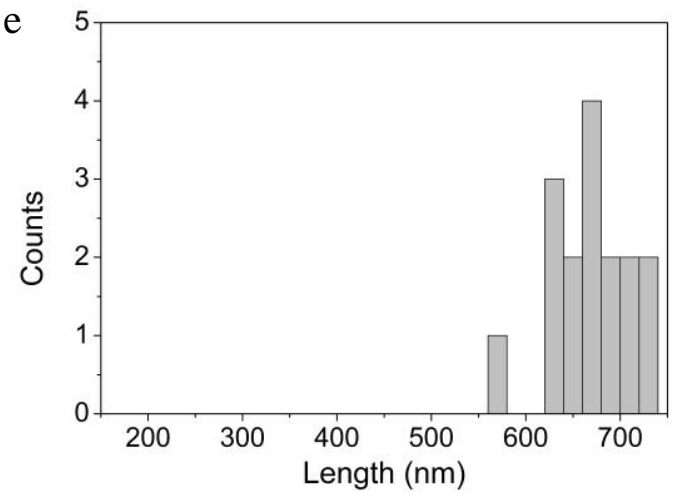

Figure S1. Length distribution histograms of the gold nanotubes embedded in AAO

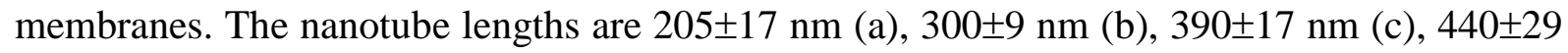
$\mathrm{nm}(\mathrm{d})$, and $670 \pm 39 \mathrm{~nm}(\mathrm{e})$, respectively, corresponding to the Au nanotubes fabricated with the electrodeposition time for $500 \mathrm{~s}, 600 \mathrm{~s}, 900 \mathrm{~s}, 1600 \mathrm{~s}$, and $1900 \mathrm{~s}$. 
a

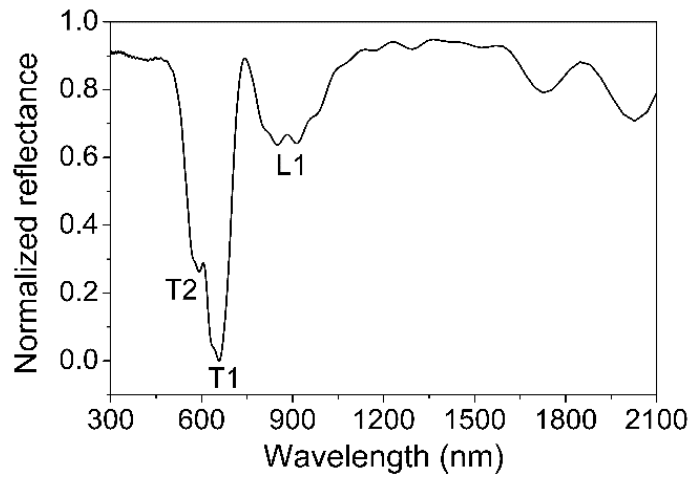

b

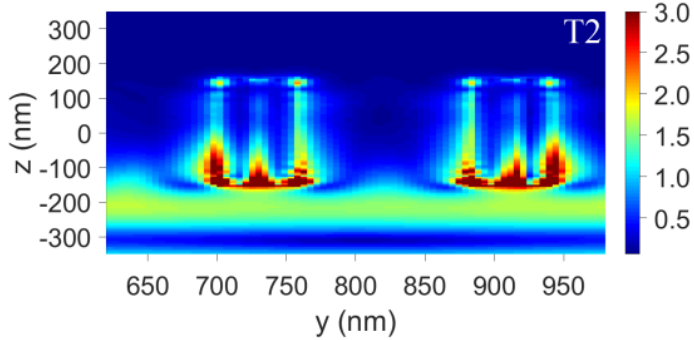

$\mathrm{c}$

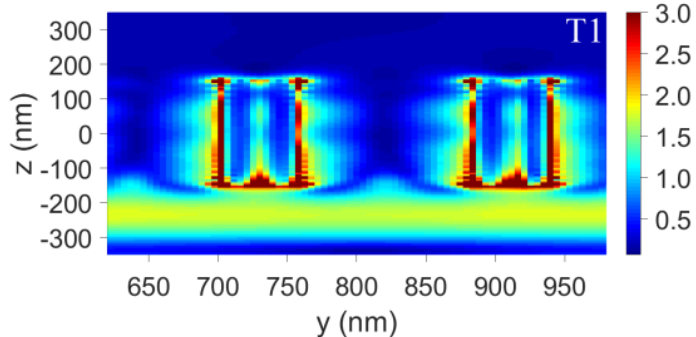

d

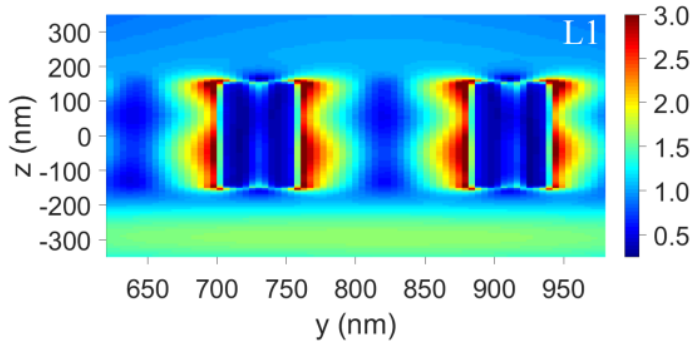

Figure S2. (a) Reflectance spectra of the gold nanotube array with the length of $300 \mathrm{~nm}$ by FDTD simulation, $(b, c)$ Corresponding to the electric field distributions of the transverse resonance modes $\mathrm{T} 2$ and $\mathrm{T} 1$ of the gold nanotube array, (d) the electric field distribution of the longitudinal resonance mode L1 of the nanotube array. 


\section{WILEY-VCH}

a

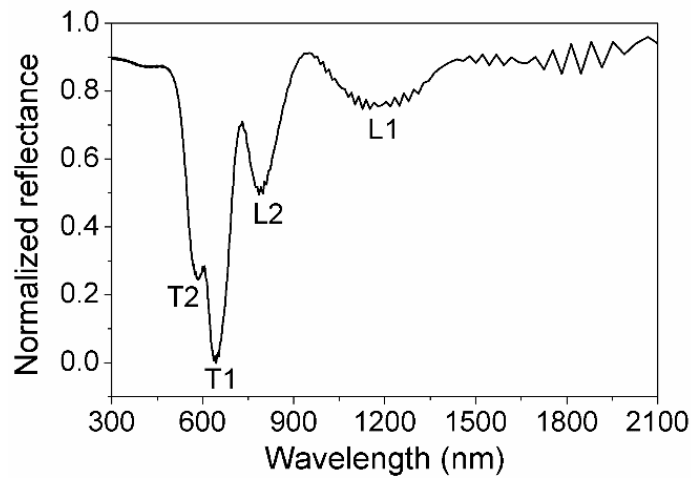

b

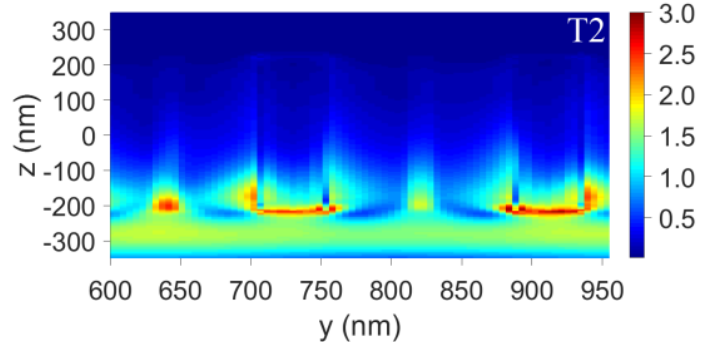

$\mathrm{c}$

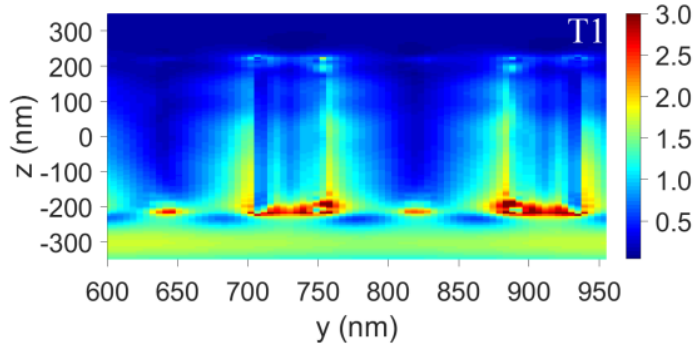

d

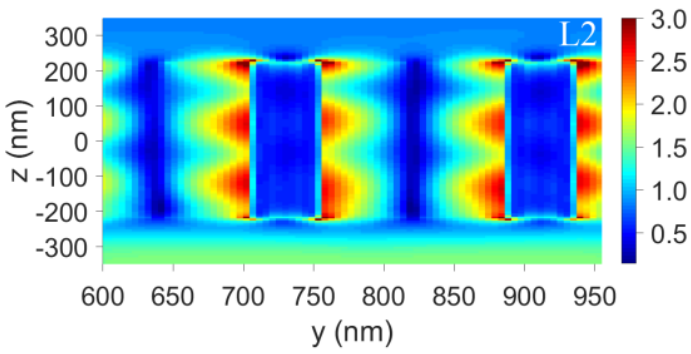

e

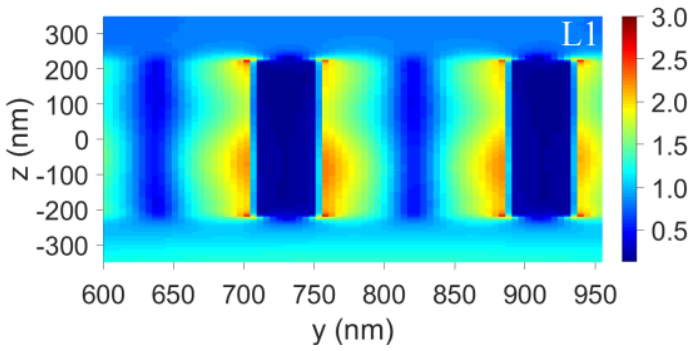

Figure S3. (a) Reflectance spectra of the gold nanotube array with the length of $440 \mathrm{~nm}$ by FDTD simulation, (b, c) Corresponding to the electric field distributions of the transverse resonance modes $\mathrm{T} 2$ and $\mathrm{T} 1,(\mathrm{~d}, \mathrm{e})$ Electric field distributions of the longitudinal resonance modes L2 and L1 with the different harmonics, respectively. 


\section{WILEY-VCH}

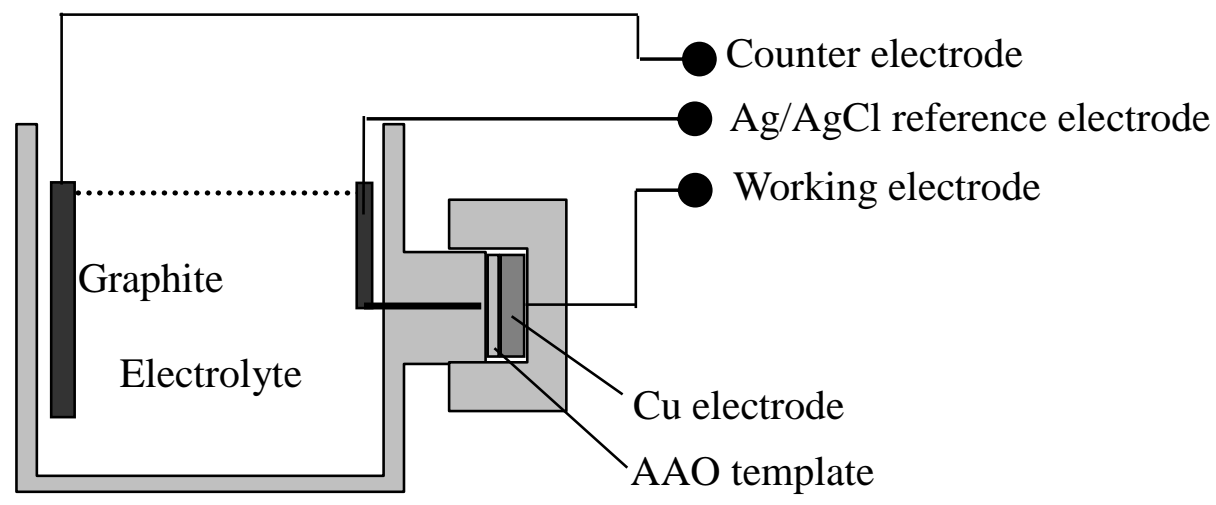

Three-electrode cell

Figure S4. Schematic illustration of three-electrode cell used for fabrication of gold nanotube arrays by using an electrochemical deposition process into the nanochannels of AAO. The experiment was performed by an electrochemical workstation (VersaSTAT 4, Princeton Applied Research (PAR)) and VersaStudio control software. 